\title{
Aspects juridiques de la radioprotection au plan international
}

\author{
J. PAPAZIAN $(*)$ \\ (Manuscrit reçu le 10 juin 1981)
}

\begin{abstract}
RÉSUMÉ
Cette note décrit les diffèrentes institutions internationales qui s'occupent de radioprotection au plan juridique et indique leurs rôles respectifs. Ces organismes sont soit non gouvernementaux (C.I.P.R., C.I.U.R.), soit gouvernementaux et alors ils peuvent intervenir au plan mondial (O.N.U., A.I.E.A., O.I.T., O.M.S.) ou au plan régional (A.E.N., O.C.D.E., EURATOM, COMECOM). La portée juridique des recommandations ou des directives qu'ils établissent est précisée.
\end{abstract}

\section{ABSTRACT}

A review is made of the respective activities of the various international organizations concerned with radiation protection at the legal level. These organizations are either non-governmental (I.C.R.P., I.C.R.U.) or governmental in which case they can act at the world (U.N., I.A.E.A., I.L.O., W.H.O.) or regional level (N.E.A., EURATOM, COMECOM). The legal impact of the recommendations or directives they issue is specified.

\section{INTRODUCTION}

Le développement de l'énergie nucléaire civile a entraîné la création d'une nouvelle branche du droit, le droit nucléaire: il concerne tous les problèmes juridiques liés aux activités de caractère nucléaire et une partie importante est consacrée à la radioprotection. D'ailleurs, la première règle juridique de radioprotection est née en $1899\left({ }^{1}\right) 4$ ans seulement après la découverte des rayons X par Röntgen.

(*) Électricité de France, Comité de Radioprotection, 39, rue de Washington, 75008 Paris.

(') Décret $\mathrm{n}^{\circ} 88311$ du 21 octobre 1899 du Gouvernement de Basse-Autriche (cf.Protection contre les rayonnements ionisants, O.M.S., Genève, 1972, p. 4).

RADIOPRUIELTION, VOL. 16 - 0033-8451/1981/243/\$ 5.00/C Bordas-Dunod. 


\section{ORGANISATIONS INTERNATIONALES NON GOUVERNEMENTALES}

\section{1. Commission internationale de Protection Radiologique (C.I.P.R.)}

Créé en 1928 par le $\mathrm{XI}^{\mathrm{e}}$ Congrès international de Radiologie, la C.I.P.R. est une organisation non gouvernementale, reconnue par les Nations Unies. Depuis 1956, elle a établi des relations officielles avec l'O.M.S. Elle a été la première institution internationale qui se soit occupée d'une façon efficace de la radioprotection.

La Commission comprend 13 membres, tous spécialistes, choisis intuitu personae, sans considération de nationalité, et 4 comités. Elle a créé aussi des groupes de travail qui peuvent faire appel à des experts.

Les fonctions de la C.I.P.R. sont purement de conseil du fait qu'il s'agit d'un organisme privé. La tâche essentielle de la Commission consiste en l'élaboration de principes fondamentaux de radioprotection, basés sur



C.I.P.R., Commission internationale de protection radiologique.

I.C.R.U., Commission internationale des unités et mesures de rayonnements.

U.N.S.C.E.A.R., Comité scientifique chargé de l'étude des effets des rayonnements ionisants.

A.I.E.A., Agence internationale de l'énergie atomique.

O.I.T., Organisation internationale du travail.

O.M.S., Organisation mondiale de la santé.

F.A.O., Organisation des Nations Unies pour l'alimentation et l'agriculture.

A.E.N., Agence pour l'énergie nucléaire.

C.E.E.A., Communauté européenne de l'énergie atomique (Euratom).

C.A.E.M., Conseil d'assistance économique mutuelle. 
l'ensemble des données scientifiques actuelles, et en la proposition des limites de doses. La C.I.P.R. formule des recommandations en toute indépendance.

\subsection{Commission Internationale des Unités et Mesures de Rayonnements} (I.C.R.U., sigle en anglais)

Cette organisation non gouvernementale émane, comme la C.I.P.R., du Congrès international de radiologie. Elle est composée de médecins et de physiciens. En application d'un accord conclu en 1955, la Commission est le principal conseiller de l'O.M.S. en matière d'unités et de mesures radiologiques et l'O.M.S. assure la diffusion de ses recommandations. Depuis 1960, I'I.C.R.U. est aussi un organisme consultatif de l'A.I.E.A.

L'I.C.R.U. et l'I.S.O. $\left({ }^{2}\right)$ émettent des recommandations favorisant l'uniformité des unités au plan international, pour éviter toute ambiguïté dans l'interprétation des limites de dose inscrites dans les normes de radioprotection ainsi que dans l'interprétation des résultats expérimentaux.

\section{ORGANISATIONS INTERNATIONALES GOUVERNEMENTALES}

\subsection{Organisation des Nations Unies (O.N.U.)}

Les activités de l'O.N.U. dans le domaine de la radioprotection s'exercent grâce à un Comité scientifique chargé de l'étude des effets des rayonnements ionisant (U.N.S.C.E.A.R.) ${ }^{3}$ ), formé uniquement de scientifiques.

L'U.N.S.C.E.A.R. publie des rapports qui sont transmis à l'Assemblée générale des Nations Unies après leur approbation à l'unanimité; ce fait est rare dans l'enceinte des Nations Unies. L'Assemblée générale de l'O.N.U. adopte les résolutions du Comité sans avoir à les voter. Bien que ce Comité n'ait pas de pouvoir normatif et que ses textes, diffusés à des fins d'information, n'aient aucune force obligatoire, le Comité jouit, au plan international, d'une grande autorité.

Une étroite et indispensable coopération le lie aux autres organismes internationaux.

\subsection{INSTITUTIONS SPÉCIALISÉES DES NATIONS UNIES}

\subsubsection{Agence internationale de l'énergie atomique (A.I.E.A.)}

L'A.I.E.A. $\left({ }^{4}\right)$ fait partie des institutions spécialisées des Nations Unies tout en étant une organisation autonome, avec des compétences se rapportant à tous les domaines de l'énergie nucléaire. Rappelons que sa mission essentielle consiste à veiller à ce que l'atome ne puisse menacer ni la paix, ni la santé.

$\left({ }^{2}\right)$ Organisation internationale de Normalisation (organisation non gouvernementale.

$\left({ }^{3}\right)$ Créé par l'Assemblée générale de l'O.N.U. le $3 / 12 / 55$, il comprend les représentants de 20 états, dont la France.

( $\left.{ }^{4}\right)$ L'Agence compte 110 États membres (fin 1980). 
Les activités de l'A.I.E.A. dans le domaine de la radioprotection sont les suivantes :

- activités normatives se rapportant aux mesures à prendre pour la protection de la santé et de la sécurité;

- action favorisant les échanges d'information et de documentation;

- action concernant les recherches, les services d'assistance technique et la formation des chercheurs;

- la conclusion d'accords de coopération et l'organisation de réunions internationales.

Aux termes de son statut, l'Agence a pour fonction d'établir des normes de sécurité destinées à protéger la santé et à réduire au minimum les dangers auxquels sont exposés les personnes et les biens. L'activité normative de l'A.I.E.A. est une de ses plus importantes fonctions statutaires. Conformément à l'article précité, l'Agence a publié, en 1962, ses premières "Normes fondamentales de radioprotection ». Elles ont été révisées en $1967\left({ }^{5}\right)$. Elles sont fondées sur les recommandations de la C.I.P.R. Ces normes et recommandations ont été acceptées par l'O.M.S. et l'O.I.T.

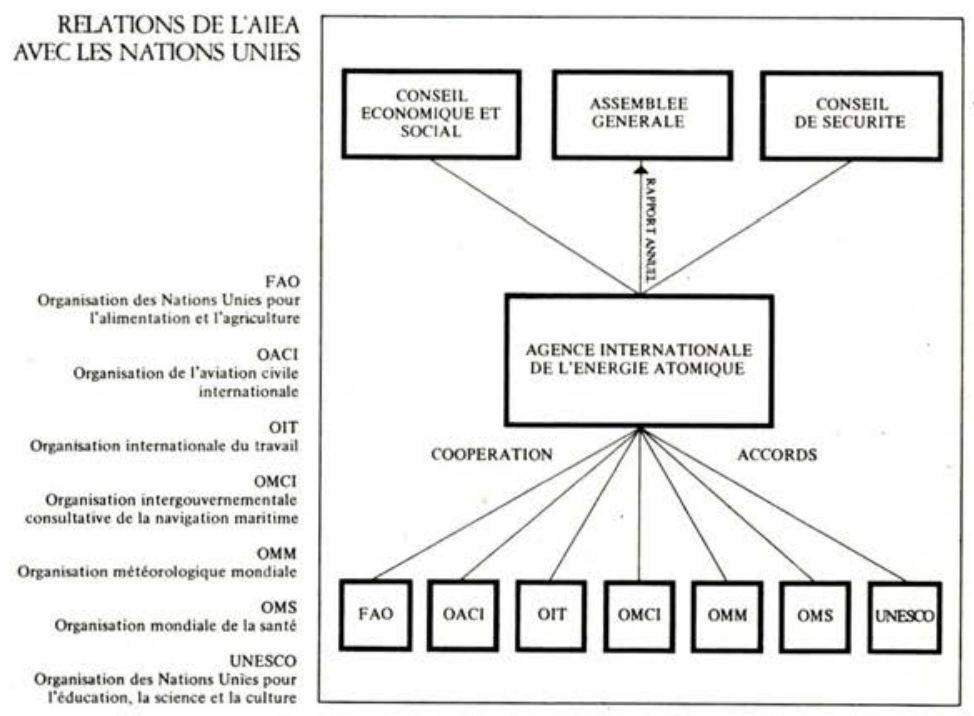

L'Agence a donc établi toute une série de normes et de recommandations; elle publie une " collection de sécurité ", des recueils, des guides et des manuels qui développent les normes fondamentales de la radioprotection et les complètent en tenant compte des règlements nationaux déjà existants, ainsi que

$\left({ }^{5}\right)$ Actuellement une révision est en cours, pour un texte unique, conjointement avec l'A.E.N., I'O.I.T. et I'O.M.S. 
des activités des organismes internationaux compétents. Les normes de sécurité et les règlements doivent être approuvées par le "Conseil des gouverneurs " avant leur publication. L'A.I.E.A. les considère comme documents de référence pouvant servir à l'établissement de règlements nationaux de santé et de sécurité.

L'A.I.E.A. a conclu plusieurs accords de coopération avec des organisations régionales et intergouvernementales, par exemple avec l'Euratom (en vigueur depuis le 1/1/1976), le Comecon, etc. (voir tableau). Ces accords ont pour but d'harmoniser les efforts de ces organisations dans la réalisation de leurs objectifs respectifs dans le domaine nucléaire.

\subsubsection{L'Organisation internationale du travail (O.I.T.)}

L'O.I.T. est une organisation intergouvernementale, qui s'efforce de promouvoir le bien-être des travailleurs.

L'O.I.T. dispose de compétence normative et élabore des mesures internationales concernant le travail et des conditions de vie des travailleurs. En matière de radioprotection, elle a adopté en 1960 la convention $\mathrm{n}^{\circ} 115$ et la recommandation $\mathrm{n}^{\circ} 114$. Avant cette date, la protection des travailleurs nucléaires faisait l'objet d'une promulgation de recommandations et de conventions destinées à tous les travailleurs. En 1934, dans la convention $\mathrm{n}^{\circ} 42$, l'O.I.T. a inclus les risques des radiations ouvrant droit à des réparations dans le tableau des maladies professionnelles. La convention $\mathrm{n}^{\circ} 115$ et la recommandation $\mathrm{n}^{\circ} 114$ s'appliquent à toutes les activités entraînant l'exposition des travailleurs à des rayonnements ionisants en mettant l'accent sur la prévention à la fois médicale et technique.

Depuis 1961, l'O.I.T. est liée à l'Euratom par un accord de coopération.

\subsubsection{Organisation mondiale de la santé (O.M.S.)}

Le but de l'O.M.S. est " d'amener tous les peuples au niveau de santé, le plus élevé possible " (article 1 de la Constitution). Pour ce qui concerne les rayonnements, l'aspect scientifique est beaucoup plus important que l'action normative. En vertu de l'article 70 de la Constitution de l'O.M.S., un accord de coopération a été signé entre l'O.M.S. et l'A.I.E.A., en 1959, pour déterminer leurs rôles respectifs. Dans le domaine nucléaire, l'Agence a un rôle prépondérant, mais en ce qui concerne le domaine de la recherche, l'O.M.S. conserve tous ses droits (article 1, $\$ 2$ de l'accord). L'O.M.S. joue le rôle de conseiller technique de l'Agence en ce qui concerne les normes de radioprotection. L'O.M.S. publie des directives et des recommandations concernant certaines utilisations des rayonnements ionisants. Elle prépare aussi avec l'A.I.E.A. des guides et des manuels sur l'application des normes.

Au siège de l'O.N.U. fonctionne un comité spécial chargé de coordonner l'activité de celle-ci avec celle des institutions spécialisées dans le domaine nucléaire. 


\title{
3. ORGANISATIONS INTERNATIONALES GOUVERNEMENTALES RÉGIONALES
}

\author{
3. 1. Agence pour L'Énergie nucléaire (A.E.N.) DE L'Organisation de \\ CoOpération et de DÉvelopPEMENT ÉCONOMIQUe (O.C.D.E.) $\left({ }^{6}\right.$ )
}

Les activités de l'O.C.D.E. en matière d'énergie nucléaire sont confiées à l'Agence pour l'énergie nucléaire - A.E.N. (l'ancienne E.N.E.A.). L'A.E.N. est un organisme autonome, fonctionnant au sein de l'O.C.D.E.

L'Agence a constitué un Comité dit de « protection radiologique et de santé publique ». Ce Comité constitue la plus haute instance technique, dans le cadre de l'A.E.N. , concernant la radioprotection. Ce Comité a les activités suivantes :

- examen critique des politiques et pratiques nationales en matière de radioprotection et de santé publique; C.I.P.R.;

- interprétation et application pratique des normes fondamentales de la

- détermination des règles fondamentales de l'A.E.N. en matière de radioprotection;

- études spécifiques des problèmes de radioprotection et d'environnement, études concernant l'exposition professionnelle des travailleurs de l'industrie nucléaire, de mines d'uranium, etc.

Ce Comité a été chargé d'élaborer des règles fondamentales en matière de radioprotection. Ainsi, en 1959, le Conseil de l'O.C.D.E. a approuvé les premières normes de base pour la protection de la santé contre les dangers des rayonnements. Ces normes ont été révisées en 1968 et en 1973; une troisième révision est en cours en collaboration avec les autres organisations intéressées, à la lumière de nouveaux principes de radioprotection exposés dans les publications $\mathrm{n}^{\circ} 22$ et 26 de la C.I.P.R. En 1960, l'A.E.N. a conclu des accords de coopération avec l'A.I.E.A. et la C.E.E. En vertu d'un protocole relatif à la Convention de l'O.C.D.E. et à l'article 16 des statuts de l'Agence, la Commission des Communautés européennes (C.E.E.) participe pleinement aux travaux de l'A.E.N.

\section{2. Euratom $\left({ }^{7}\right)$}

C'est une organisation régionale créée en 1957 par le Traité de Rome. L'article 2 du traité défınit ses tâches. Le Traité de Rome a donné une place importante aux problèmes de radioprotection, en prévoyant une action normative.

( $\left.{ }^{6}\right)$ Membres de I'O.C.D.E. : Allemagne Fédérale, Australie, Autriche, Belgique, Canada, Danemark, Espagne, États-Unis, Finlande, France, Grèce, Irlande, Islande, Italie, Japon, Luxembourg, Norvège, Nouvelle-Zélande, Pays-Bas, Portugal, Royaume-Uni, Suède, Suisse et Turquie.

(7) Membres de la Communauté européenne : Allemagne Fédérale, Belgique, Danemark, France, Grèce, Irlande, Italie, Luxembourg, Pays-Bas et Royaume-Uni. 
Conformément aux dispositions du chapitre III du Traité, le Conseil des ministres a adopté, en 1959, des directives établissant des normes de base relatives à la protection sanitaire de la population et des travailleurs contre les dangers des rayonnements ionisants. Ces normes ont été amendées en 1962, 1966, 1976 et plus récemment en 1979 (cf. directive $\mathrm{n}^{\circ} 79 / 343$ du 3 avril 1979) et en 1980 (directive du 15 juillet 1980/ 80/836 Euratom). La Commission européenne ne se limite pas à élaborer des normes, mais elle surveille aussi leur application dans les législations nationales. Ainsi, grâce aux pouvoirs étendus que lui confère le Traité (articles 2, 30, 37 et 39) l'Euratom a pu réaliser une politique de radioprotection commune et harmonisée, notamment en matière de réglementation communautaire.

L'Euratom a aussi son propre programme de recherche en matière de radioprotection. Le nouveau programme 1980-1984 comporte six activités : la dosimétrie, le contrôle, les effets somatiques à court et à long terme, les effets génétiques des rayonnements et l'évaluation des risques d'irradiation.

\section{3. Conseil d’Assistance Économique mutuelle (COMECOM)}

Dans le cadre du Comecon $\left({ }^{8}\right)$, il a été créé une commission permanente des utilisations pacifiques de l'énergie atomique. Cette Commission s'occupe aussi de la radioprotection en instituant une coopération pour l'élaboration et la défınition des normes et pour les aspects médicaux de la sécurité radiologique.

En 1965-1966, la Commission a publié aussi ses propres normes de radioprotection. En grande partie, ces normes tenaient compte des recommandations de la C.I.P.R., avec cette particularité qu'elles étaient encore plus restrictives et plus prudentes. Deux ans plus tard, ces normes ont cessé d'être appliquées, le Conseil ayant recommandé à ses États membres d'observer les normes de la C.I.P.R. et de l'A.I.E.A. Depuis, la Commission a continué à donner des recommandations selon les besoins.

\section{LA PORTÉE JURIDIQUE DES NORMES INTERNATIONALES}

Les moyens juridiques des organisations internationales et leur portée juridique sont déterminés dans les actes constitutifs de chaque organisation.

En matière de protection radiologique, nous nous trouvons actuellement en présence de plusieurs instruments juridiques tels que les recommandations, les conventions et les directives.

La recommandation est une forme d'expression du droit qui ne possède aucune force contraignante, elle n'a pas de pouvoir réglementaire propre et sa non-application n'entraîne aucune sanction. Malgré sa valeur juridique limitée, elle reste un des instruments juridiques le plus utilisé par les organismes internationaux. Ces derniers adressent des recommandations aux États ou aux autres organisations internationales.

$\left({ }^{8}\right)$ Conseil d'assistance économique mutuelle pour les pays socialistes.

VOL. $16-\mathrm{N}^{\circ} 4$ 


\section{1. Les ReCOMmandations DE LA C.I.P.R.}

Les recommandations de la C.I.P.R., sans avoir force obligatoire, exercent une influence sur l'élaboration des normes juridiques par les autres organisations internationales gouvernementales, en raison de leur valeur scientifique et technique. Ces recommandations ont un caractère doctrinal et non réglementaire, c'est la raison pour laquelle elle ne peuvent pas prendre une forme réglementaire reproductible directement dans les législations nationales.

La Commission publie parfois aussi des rapports. Un rapport de la C.I.P.R. n'est pas créateur de droit non plus, mais il possède une indéniable valeur incitative.

\section{2 LA PORTÉE JURIDIQUE DES NORMES DE L'A.I.E.A.}

Par contre, les normes de l'A.I.E.A. peuvent posséder une force obligatoire. Cette force varie selon les cas. En général, l'Agence n'a pas de compétence réglementaire s'imposant aux États membres. Elle ne peut que leur recommander ses normes à titre de modèle.

Toutefois, les normes de l'A.I.E.A. peuvent devenir obligatoires pour les États concernés dans deux cas précis : d'une part, après accords bilatéraux ou multılatéraux qui lui prêtent ce pouvoir et, d'autre part, quand les normes sont appliquées directement aux opérations menées par l'Agence même, ainsi qu'aux opérations concernant des États tiers ayant sollicité une assistance fournie par l'A.I.E.A.

\section{3. LA CONVENTION ET LA RECOMMANDATION DE L'O.I.T.}

La convention est un instrument juridique qui déclenche une action législative. Elle constitue, en effet, un accord, créateur d'obligations internationales pour le pays intéressé. C'est pourquoi, la convention exige une ratification $\left({ }^{9}\right)$, alors que la recommandation n'en a pas besoin. Les États s'engagent à soumettre, dans un délai d'un an et jamais de plus de 18 mois, ces deux instruments aux autorités nationales compétentes en vue de les faire transformer en loi nationale ou de leur faire prendre des mesures d'un autre ordre, inspirées de l'esprit inscrit dans l'un ou l'autre de ces actes. L'État doit, en effet, rendre compte de l'action qu'il aura exercée pour appliquer la convention, après sa ratification; le cas échéant, il expliquera et justifiera son inaction. La recommandation, par contre, ne peut donner lieu à aucune obligation de ce genre, l'État s'engage seulement à prendre en considération, mais non pas à adopter. La recommandation est donc destinée à orienter l'action sur le plan national. Après la ratification de la convention, le gouvernement concerné est tenu de présenter, périodiquement, un rapport sur les dispositions qui ont été prises pour lui donner effet.

L'O.I.T élabore aussi des règlements-type lesquels n'impliquent cependant

( ${ }^{9}$ La France a ratifié la convention $\mathrm{n}^{\circ} 115$ de l'O.I.T. concernant la radioprotection le 18 novembre 1971 . 
aucune oblıgation pour quiconque. Ils ont simplement pour objet d'aider les gouvernements dans la rédaction ou la révision de leurs propres règlements nationaux.

La constitution de l'O.I.T. ( $c f$. article 24-34), a prévu et réglé une procédure détaillée, permettant la prise à partie d'un État membre qui n'aurait pas assuré d'une manière satisfaisante l'exécution d'une convention par lui ratifiée ou qui aurait mal appliqué une recommandation.

\section{4. LES NORMES DE L'O.C.D.E./A.E.N.}

En ce qui concerne la portée juridique des normes de l'O.C.D.E., elles sont communiquées aux États membres sous la forme d'une simple recommandation. Les recommandations sont soumises à l'approbation des membres afın que ceux-ci les prennent effectivement en considération et les mettent à exécution, s'ils l'estiment opportun. Dans le domaine de la radioprotection, les décisions obligatoires sont exceptionnelles, la règle générale veut qu'un gouvernement puisse s'abstenir sans faire obstacle à la décision.

Selon l'article 11 des statuts de l'A.E.N., les décisions, avis ou recommandations du Comité de direction sont adoptés par accord mutuel des membres présents et votants et les décisions engageant les gouvernements n'obligent que les pays qui les ont acceptées $\left({ }^{10}\right)$. Compte tenu de la souplesse des mécanismes utilisés par l'O.C.D.E., les modalités de mise en œuvre sont laissées à l'initiative des autorités nationales.

L'A.E.N. prévoit une étroite collaboration avec l'A.I.E.A. et la C.E.E., afın d'harmoniser les dispositions législatives et réglementaires nationales.

\section{5. Les diRECTIVES de L'EuRATOM}

La base et la valeur juridique des normes de l'Euratom sont fixées par le Traité de Rome (1957). En vertu de ce traité, la Commission des Communautés européennes a des pouvoirs réglementaires dans le domaine de la radioprotection, plus étendus que les autres organisations internationales étudiées. L'instrument juridique qui fixe les normes est la directive. Elle a pour objectif le rapprochement des législations des pays membres. Les directives et les recommandations de la C.C.E. concernant la radioprotection représentent les bases d'une action normative qui ne trouve son équivalent dans aucune autre institution internationale. La directive indique aux États membres le but ultime à atteindre. Chaque État membre est obligé d'établir des dispositions législatives, réglementaires et administratives nationales qui permettent d'assurer le respect des normes de base fixées; l'État n'a donc le choix que de la forme et des moyens. Les États membres communiquent à la Commission toutes les dispositions prises en application de la directive.

Actuellement, la France dispose d'un délai de 30 mois pour prendre les mesures nécessaires pour se conformer à la directive du 15 juillet 1980 qu'elle doit appliquer en tant que signataire du Traité de Rome.

$\left({ }^{10}\right)$ La France a approuvé la décision concernant les normes de base dès 1959.

VOL. $16-\mathrm{N}^{\circ} 4$ 


\section{CONCLUSION}

On a vu que chaque organisation internationale a édicté sa propre réglementation en matière de radioprotection. Ceci peut faire craindre que des contradictions ne se produisent au niveau des textes et de leur application. Ce n'est heureusement pas le cas, bien au contraire.

Le régime juridique applicable en la matière est, en effet, caractérisé par un très haut degré d'unification et de coordination au niveau international. Malgré la diversité des organismes internationaux, il n'y a finalement pas de divergence en matière de normes. Cette situation résulte de l'action de la C.I.P.R. et de ses recommandations qui ont servi de base à l'élaboration des normes par les autres organisations internationales. Ce phénomène juridique, exceptionnel, permet d'éviter certains conflits.

Il faut également souligner l'effort incontestable d'uniformisation qui permet de rapprocher le point de vue des scientifiques et des juristes, des chefs d'entreprises et des salariés (par l'intermédiaire de l'Organisation internationale du travail), des économistes, etc.

Depuis 1977, l'A.I.E.A., l'A.E.N., l'Q.I.T. et l'O.M.S. ont décidé de réviser conjointement les normes actuelles sur la radioprotection et de disposer à l'avenir d'un texte unique commun. Cette révision est en cours.

\section{ANNEXE}

\section{AdRESSES DES ORGaNISATIONS INTERNATIONALES}

O.N.U., Organisation des Nations Unies, United Nations Headquarters, New York, N.Y. 10017 (U.S.A.). Palais des Nations, 1211 Genève 10 (Suisse).

O.I.T., Organisation internationale du travail, 4, route des Morillons, 1211 Genève 22 (Suisse).

O.M.S., Organisation mondiale de la santé, 1211 Genève 27 (Suisse).

A.I.E.A., Agence internationale de l'énergie atomique, 20, Wagramerstrasse 5, P.O. Box 100, A-1400 Vienne (Autriche).

A.E.N./O.C.D.E., Agence pour l'énergie nucléaire de l'O.C.D.E., 2, rue André-Pascal, 75775 Paris Cedex 16 (France).

C.I.P.R. ou I.C.R.P., Commission internationale de protection radiologique, International Commission on Radiological Protection, Dr F. D. Sowby (Scientific secretary), Clifton Avenue, Sutton, Surrey SM 2 SPU (GrandeBretagne).

I.C.R.U., International Commission on Radiation Units and Measurements, 7910 Woodmont Av., Washington DC 2014 (U.S.A.).

C.C.E., Commission des Communautés européennes (Euratom), rue de la Loi, 200 B, 1049 Bruxelles (Belgique).

\section{Remerciements}

Ce texte a été revu par M. J. HéBerT, chef de la Division Droit nucléaire et Droit international, de la Direction de l'Équipement, E.D.F., que nous remercions vivement. 


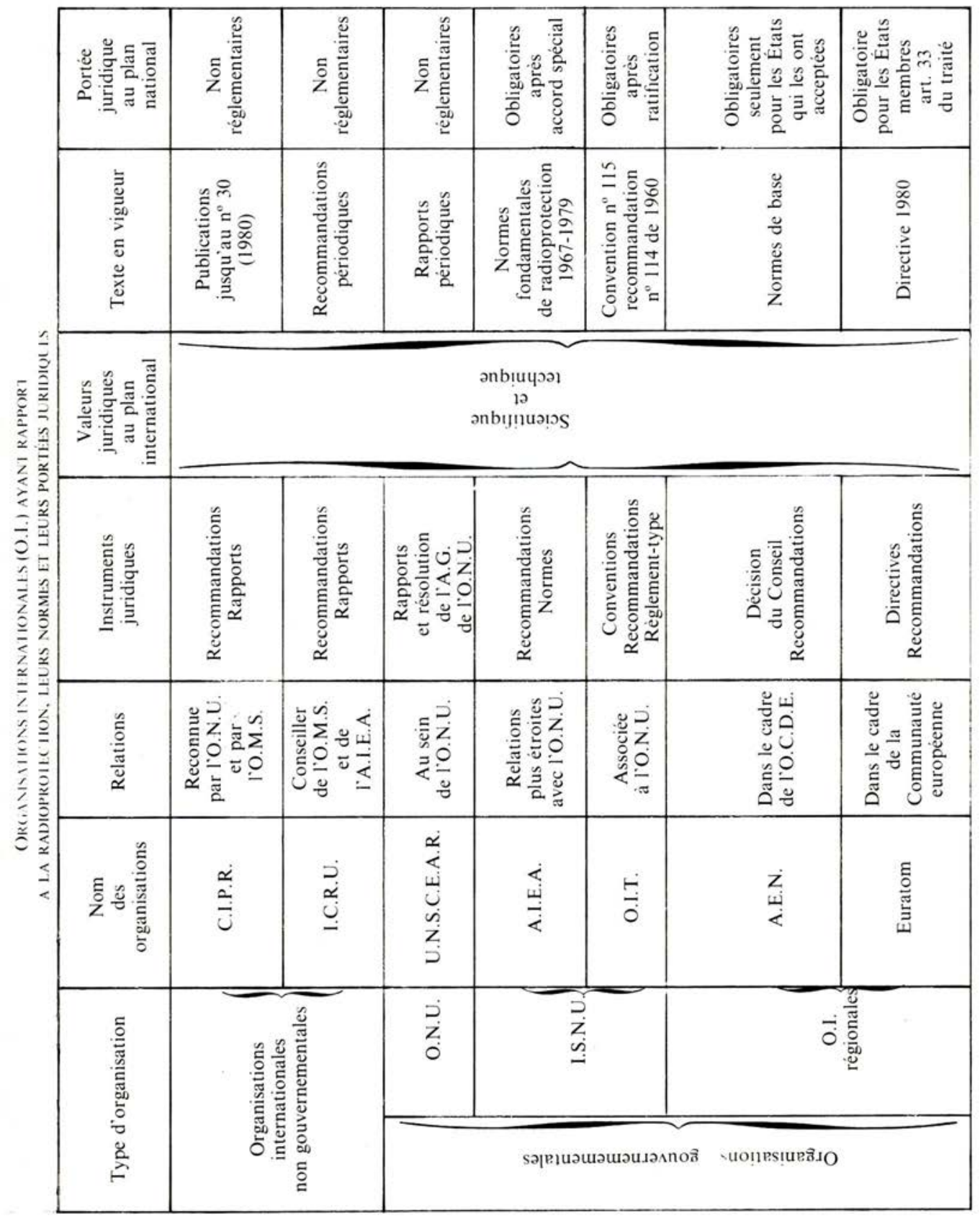

vol. $16, \mathrm{n}^{\circ} 4$ 